\title{
Controlling domain wall pinning in planar nanowires by selecting domain wall type and its application in a memory concept
}

D. Atkinson, D. S. Eastwood, and L. K. Bogart

Citation: Appl. Phys. Lett. 92, 022510 (2008);

View online: https://doi.org/10.1063/1.2832771

View Table of Contents: http://aip.scitation.org/toc/apl/92/2

Published by the American Institute of Physics

\section{Articles you may be interested in}

The design and verification of MuMax3

AIP Advances 4, 107133 (2014); 10.1063/1.4899186

The effect of geometrical confinement and chirality on domain wall pinning behavior in planar nanowires Journal of Applied Physics 104, 033904 (2008); 10.1063/1.2961313

Field- and current-induced domain-wall motion in permalloy nanowires with magnetic soft spots Applied Physics Letters 98, 202501 (2011); 10.1063/1.3590267

Domain wall pinning and potential landscapes created by constrictions and protrusions in ferromagnetic nanowires

Journal of Applied Physics 103, 114307 (2008); 10.1063/1.2936981

Direct observation of domain-wall pinning at nanoscale constrictions

Applied Physics Letters 87, 102509 (2005); 10.1063/1.2042542

Domain wall pinning for racetrack memory using exchange bias

Applied Physics Letters 105, 162406 (2014); 10.1063/1.4899134

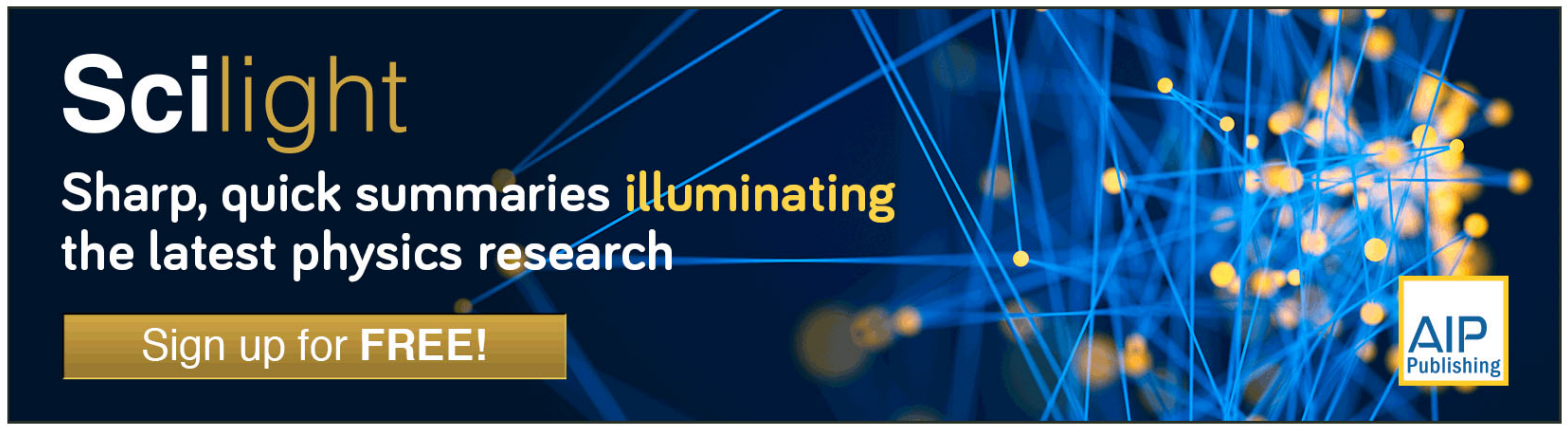




\title{
Controlling domain wall pinning in planar nanowires by selecting domain wall type and its application in a memory concept
}

\author{
D. Atkinson, a) D. S. Eastwood, and L. K. Bogart \\ Department of Physics, Durham University, DH1 3 LE, United Kingdom
}

(Received 12 December 2007; accepted 17 December 2007; published online 16 January 2008)

\begin{abstract}
Here, we report on the control of domain wall pinning at notch features patterned in Permalloy planar nanowires by selecting the micromagnetic configuration of the domain wall using a transverse magnetic field. The domain wall behavior was investigated both experimentally using focused magneto-optic Kerr effect measurements of lithographically patterned nanowires and with micromagnetic simulations. The pinning behavior observed is utilized in a concept for multibit memory cells applicable as the free layer in magnetic random access memory where the domain structure is defined by the location of domain walls that either pin or passby pinning structures depending upon the domain wall configuration selected. (C) 2008 American Institute of Physics.
\end{abstract} [DOI: $10.1063 / 1.2832771]$

There has been an intensive research interest in the behavior of individual domain walls in nanowires both in terms of the fundamental physics ${ }^{1}$ and for potential applications in sensors, ${ }^{2}$ logic, ${ }^{3,4}$ and memory. For memory applications, domain walls can be pinned at structural features such as notches creating multiple domains and hence multiple memory states which could be read out either sequentially in shift registers ${ }^{3,5}$ or directly if an entire nanowire forms the free layer of a magnetic random access memory (MRAM) cell. ${ }^{6}$ In contrast to conventional single bit per transistor MRAM, this approach presents the opportunity to store multiple data bits for each readout transistor in the associated complementary metal-oxide semiconductor circuitry. Here, we investigate controlling the interactions of domain walls with asymmetric pinning structures by selecting the magnetization state of the domain wall using a small magnetic field applied transverse to the wire axis. This provides an additional degree of freedom to the control the pinning and may be exploited in future magnetic memory.

In polycrystalline Permalloy (nominally $\mathrm{Ni}_{81} \mathrm{Fe}_{19}$ ) nanowires, the weak magnetocrystalline anisotropy and small magnetostriction allow the magnetization to be largely constrained by magnetostatic energy considerations so as to lie along the long axis of the wire with spins parallel to the surfaces and edges. When structural features such as notches or protrusions are patterned as part of the nanowire, the spin structure will tend to follow the local edges of these features. The micromagnetic structure of domain walls in planar nanowires depends upon the thickness and width of the wire ${ }^{7}$ and varies from transverse walls in narrower/thinner wires through an asymmetric transverse wall structure to vortex walls for wider and thicker nanowires. ${ }^{8}$ The micromagnetic structure of the walls can have more than one orientation. For transverse walls, the spins are largely in plane and at the center of the wall the spin points in one of two directions at $90^{\circ}$ to the wire axis, while for vortex walls the sense of rotation of the spin structure can be clockwise or counterclockwise. In a symmetrical nanowire, these different configurations are energetically equivalent and symmetrical features, such as paired notches located on opposite sides of a nanowire, present the same energy barrier to both possible

\footnotetext{
${ }^{a)}$ Electronic mail: del.atkinson@durham.ac.uk.
}

magnetization configurations of transverse walls. In contrast, it is suggested that the spin configuration through a structurally asymmetric feature, such as a triangular notch in only one side of a nanowire, will present different energetic barriers to propagating domain walls depending upon both the spin structure of the wall and whether the wall separates head-to-head or tail-to-tail oriented domains. It is expected that this energetic difference would be manifested as a difference in the field required to depin a domain wall depending upon the direction the domain wall magnetization and whether it separates head-to-head or tail-to-tail domains. The concept of controlling the domain wall pinning by setting the wall magnetization state is investigated here using micromagnetic simulations and experimental measurements of individual planar nanowires.

Micromagnetic simulations were performed using the OOMMF package developed by NIST. ${ }^{9}$ Domain wall pinning behavior was simulated for a planar nanowire with a staircased triangular notch of depth $176 \mathrm{~nm}$ into the wire located part way along the length of the wire. At one end, the wire is terminated with a wider "nucleation pad" and with triangular point at the other end. The modeled nanowire was $496 \mathrm{~nm}$ wide and $8 \mathrm{~nm}$ thick and the nucleation pad was $1496 \mathrm{~nm}$ wide, see Fig. 1. The simulation used the standard magnetic parameters for Permalloy, a cell size of $8 \mathrm{~nm}$ and a damping factor $\alpha$ of 0.015 .

Planar magnetic nanowires were fabricated by electron beam lithographic patterning using a single layer polymethyl methacrylate resist followed by thin-film deposition and liftoff. Thin films were deposited by thermal evaporation at a pressure of $10^{-7}$ Torr and a rate of $\sim 1 \AA$ /s from a single $\mathrm{Ni}_{81} \mathrm{Fe}_{19}$ source. The nanowire thickness was $5 \mathrm{~nm}$ as determined by an in situ deposition rate monitor that was calibrated to film thicknesses determined by $\mathrm{x}$-ray reflectivity measurements. Nanowires were $50 \mu \mathrm{m}$ long and terminated at one with a wider rectangular nucleation pad to generate domain walls at one end and a triangular point at the other end to prevent nucleation of domain walls. A single triangular notch was located halfway along the length of the nanowire.

The magnetization behavior of individual nanowires was measured using a longitudinal magneto-optic Kerr effect (MOKE) magnetometer where the focused laser spot width 


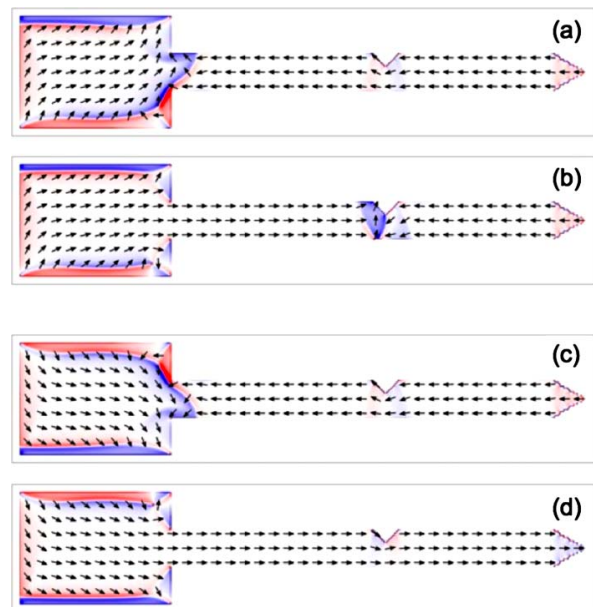

FIG. 1. (Color online) Micromagnetic simulations showing the behavior of domain walls with different magnetization configurations. (a) The formation of a head-to-head transverse wall with magnetization pointing upwards and (b) pinning of the wall at the notch after injection of the wall from the pad at 45 Oe. (c) The formation of a head-to-head transverse wall with magnetization pointing downward and (d) the domain structure at 45 Oe after injection of the wall from the pad, the wall has propagated past the notch and swept out of the wire.

on the sample was $5 \mu \mathrm{m}$. The nanowires were designed to be long enough such that the regions of the nanowire before and after the pinning structure could be interrogated independently. The MOKE measurements were made with an alternating field at $27 \mathrm{~Hz}$ and a small static transverse field applied. Care was taken to measure and account for the very small component of axial magnetic field generated by the transverse field coils, as this could cause a shift of the axial field hysteresis loops.

Figure 1 shows some examples from the micromagnetic simulations of domain wall interactions with an asymmetric pinning structure (that is only on one side of the nanowire). In Fig. 1(a), a domain wall is generated by an axial field by reversal of the wider nucleation pad structure with domain wall magnetization directed upward (toward the side with the notch). In this case, the wall has a transverse structure and separates head-to-head domain magnetization. The direction of the wall moments is controlled by the application of a small (5 Oe) magnetic field transverse to the long axis of the nanowire during the domain wall formation. With increasing field the domain wall is depinned from the pad at 45 Oe and propagates along the wire and becomes pinned at the notch, Fig. 1(b). The wall remains pinned until the axial field reached 60 Oe when it propagates along the remainder of the wire to fully reverse the structure. In contrast, when a transverse head-to-head wall is formed with a wall magnetization in the opposite (downward) direction, Fig. 1(c), the wall also depins from the pad at $45 \mathrm{Oe}$ as for the other wall orientation, but at 45 Oe the wall propagates along the whole length of the wire and is not pinned at the notch, Fig. 1(d). If this wall was depinning from the pad at a lower field it may be expected that the wall would pin at the notch at some field below 45 Oe. A clear difference is observed between the pinning potential imposed by the notch that depends upon the sense of the wall magnetization. This difference also depends upon the sense of the magnetization with head-to-head and tail-to-tail walls responding in opposite ways because of the spin structure presented at the notch by the domains and the wall. The important point to note is that the pinning strength

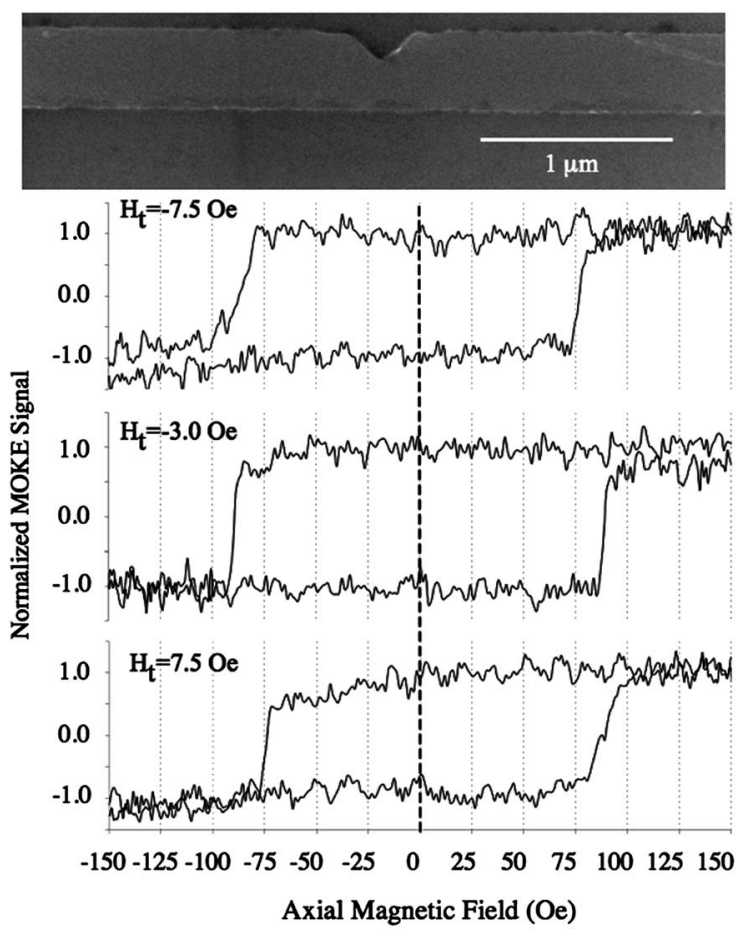

FIG. 2. Scanning electron micrograph example of a triangular notch structure in a planar nanowire and MOKE hysteresis loops measured with the laser spot located after the notch-the static transverse fields applied during the measurements are indicated.

at the notch is high when the spins in the wall oppose the direction of the magnetostatically determined spins around the notch and low when the spins in the wall and the spins structure around notch are in the same direction.

The dependence of pinning behavior upon the sense of the domain wall magnetization was investigated experimentally. Figure 2 shows an example scanning electron microscopy image of a single notch structure. Magnetization switching behavior was measured at various places along the nanowires by positioning the focused spot laser of the magneto-optic kerr effect measurement system. The switching behavior of a $220 \mathrm{~nm}$ wide, $5 \mathrm{~nm}$ thick Permalloy (nominally $\mathrm{Ni}_{81} \mathrm{Fe}_{19}$ ) nanowire with a triangular notch $60 \mathrm{~nm}$ deep halfway along the length of the wire is described here. First, with no transverse field applied measurements showed the large nucleation pad switched at about 6 Oe and domain walls were injected into the nanowire at $50 \mathrm{Oe}$. Measurements of magnetization switching past the notch showed that the depinning field was around 90 Oe and that the switching field was the same magnitude for both orientations of magnetization reversal. In order to control the formation of a specific direction of domain wall magnetization, a small amplitude static magnetic field was applied in the plane and transverse to the wire axis during the axial MOKE measurements. The influence of the transverse field upon the domain wall formation was observed in measurements of the nucleation pad where the switching field was observed to decrease as a function of increasing transverse field, this can be understood as the shape anisotropy of the pad is relatively low and the magnetic moments are rotated by the transverse field and therefore the torque from the axial field is larger leading to lower fields to initiate reversal. The influence of the transverse field was also observed for the injection of the domain wall from the nucleation pad into the nanowire. For a given direction of the transverse field, the domain wall injection 


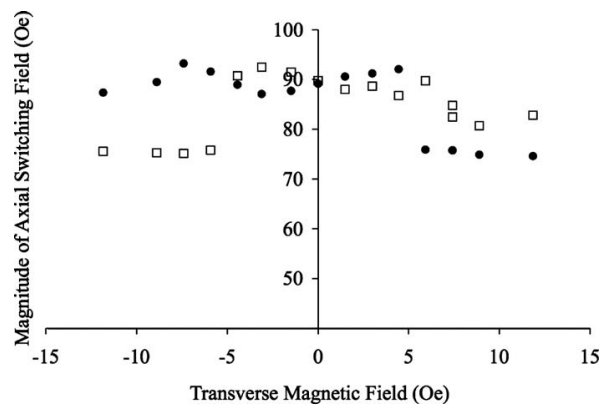

FIG. 3. The effect of transverse field upon the magnitude of the axial depinning field from the notch for head-to-head (solid symbols) and tail-to-tail (open symbols) domain structures.

field for switching from one magnetization direction to the other (negative to positive magnetization) was increased by 8 Oe and reduced only a couple of oersteds for magnetization switching in the opposite direction. This behavior was reversed when the transverse field was in the opposite direction with an increased switching field for switching from positive to negative magnetization directions. This behavior is consistent with symmetry breaking of the pinning energy states at the pad-wire interface of the domain walls that depends upon the direction of the domain wall magnetization in the presence of the transverse field. ${ }^{10}$

The primary interest here is the influence that the wall magnetization direction has upon the interaction between the wall and the asymmetrical pinning structure, in this case, a triangular notch. Figure 2 shows magnetization switching behavior measured with the MOKE laser spot located after the notch and therefore shows the switching behavior resulting from depinning of domain wall from the notch. For transverse fields below $\pm 5 \mathrm{Oe}$, the switching depinning field showed only some small changes; however, above \pm 5 Oe, the axial switching behavior changed significantly. For positive transverse fields greater than $5 \mathrm{Oe}$, the negative switching field was reduced while the positive switching field remained the same, and for negative transverse fields, the opposite behavior was observed with the positive switching field reduced and the negative switching field largely unchanged. These changes in switching field are summarized in Fig. 3 where the magnitude of the switching fields are plotted as a function of the transverse field. The data indicate that above 5 Oe the transverse field is sufficient to consistently set the magnetization direction of the domain wall that is formed by reversal of the nucleation pad. Figure 3 shows that the depinning field can be selected to be low or high by setting the orientation of the domain wall magnetization by applying a transverse field during the domain wall nucleation process. The transverse field sets the wall magnetization in the same transverse direction for both head-to-head and tailto-tail domain configurations leading to different domain wall energy states at the notch resulting from the direction of the wall magnetization with respect to the preferred orientation of the domain magnetization around the notch. Therefore, consistent with the micromagnetic simulations, it is suggested that for a head-to-head domain configuration a low pinning field is achieved for wall magnetization that is pointing away from the apex of the notch, while for a tail-to-tail configuration a low pinning field is achieved when the wall magnetization is directed toward the apex of the notch. This selectivity is expected also to be applicable to vortex domain

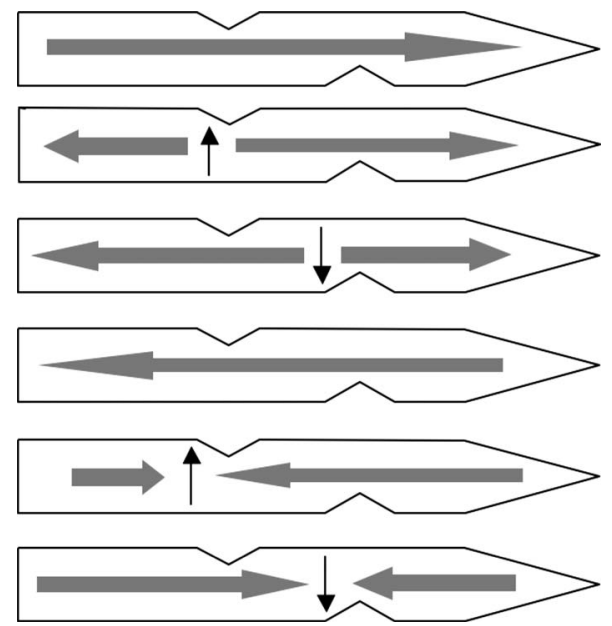

FIG. 4. A concept for a multibit memory cell. In addition to the two oppositely magnetized saturated states, further states can be obtained depending upon the direction of magnetization and the pinning location of the domain wall which is determined by the orientation of the wall magnetization (indicated by the vertical arrows) that has been selected.

walls as different wall chiralities will lead to different energy states at the pinning structure.

The control over domain wall pinning at asymmetric features described here provides an additional degree of freedom for controlling domain wall position in potential applications. Figure 4 outlines a nanowire memory cell concept that takes advantage of this additional degree of freedom to control the domain wall location and hence the magnetization configuration that can represent a memory state. The simplest memory structure consists of a nanowire with asymmetric notches located at different positions along the length of the wire as shown. Domain walls are formed at one end by an axial field and propagate along the wire to pass or become pinned by the notches depending upon the magnetization direction of the wall. Simply by selecting the direction of the wall magnetization with a small transverse field, the location at which the wall is pinned is controlled without having to change the magnitude of the axial magnetic field. Figure 4 shows that six different magnetic domain configurations are possible for this structure and each of these can represent a different memory state. Further domain configurations are possible with additional pinning sites that have higher pinning fields. By integrating such a nanowire as the free layer in magnetic tunnel junction, the memory states could be read out as a set of different resistance states of the junction. This would provide an enhanced data storage density because multiple bits can be stored for a single readout transistor.

${ }^{1}$ C. H. Marrows, Adv. Phys. 54, 585 (2005).

${ }^{2}$ M. Diegel, R. Mattheis, and E. Halder, IEEE Trans. Magn. 40, 2655 (2004).

${ }^{3}$ D. A. Allwood, G. Xiong, M. D. Cooke, C. C. Faulkner, D. Atkinson, N. Vernier, and R. P. Cowburn, Science 296, 2003 (2002).

${ }^{4}$ D. A. Allwood, G. Xiong,. C. Faulkner, D. Atkinson, D. Petit, and R. P. Cowburn, Science 309, 1688 (2005).

${ }^{5}$ S. S. P. Parkin, U.S. Patent No. 6,834,005 (December 21, 2004).

${ }^{6}$ J. Nickel and M. K. Bhattacharyya, U.S. Patent No. 7,078,244 (July 18, 2006).

${ }^{7}$ R. D. McMichael and M. J. Donahue, IEEE Trans. Magn. 33, 4167 (1997).

${ }^{8}$ Y. Nakatani, A. Thiaville, and J. Miltat, J. Magn. Magn. Mater. 290-291, 750 (2005).

${ }^{9}$ http://math.nist.gov/oommf/

${ }^{10}$ M. T. Bryan, D. Atkinson, and D. A. Allwood, Appl. Phys. Lett. 88, 032505 (2006) 